\title{
Cardiotoxicity of Anticancer Therapeutics
}

\author{
Jerry Dong ${ }^{1,2,3}$ and Hong Chen ${ }^{1,3 *}$ \\ ${ }^{1}$ Cardiovascular Biology Program, Oklahoma Medical Research Foundation, Oklahoma City, OK, United States, ${ }^{2}$ Case \\ Western Reserve University, Cleveland, $\mathrm{OH}$, United States, ${ }^{3}$ Department of Surgery, Vascular Biology Program, Boston \\ Children's Hospital, Harvard Medical School, Boston, MA, United States
}

OPEN ACCESS

Edited by:

Jun-ichi Abe,

University of Texas MD Anderson Cancer Center, United States

Reviewed by:

Scott James Cameron,

University of Rochester,

United States

Francis Kim,

University of Washington,

United States

Anecita P. Fadol,

University of Texas MD Anderson

Cancer Center, United States

*Correspondence:

Hong Chen

hong.chen@childrens.harvard.edu

Specialty section:

This article was submitted to

Atherosclerosis and Vascular

Medicine,

a section of the journal Frontiers

in Cardiovascular Medicine

Received: 06 September 2017

Accepted: 22 January 2018

Published: 07 February 2018

Citation:

Dong $J$ and Chen H (2018)

Cardiotoxicity of Anticancer

Therapeutics.

Front. Cardiovasc. Med. 5:9. doi: 10.3389/fcrm.2018.00009
As cancer therapeutics continues to improve and progress, the adverse side effects associated with anticancer treatments have also attracted more attention and have become extensively explored. Consequently, the importance of posttreatment follow-ups is becoming increasingly relevant to the discussion. Contemporary treatment methods, such as tyrosine kinase inhibitors, anthracycline chemotherapy, and immunotherapy regimens are effective in treating different modalities of cancers; however, these reagents act through interference with DNA replication or prevent DNA repair, causing endothelial dysfunction, generating reactive oxygen species, or eliciting non-specific immune responses. Therefore, cardiotoxic effects, such as hypertension, heart failure, and left ventricular dysfunction, arise posttreatment. Rising awareness of cardiovascular complications has led to meticulous attention for the evolution of treatment strategies and carefully monitoring between enhanced treatment effectiveness and minimization of adverse toxicity to the cardiovasculature, in which psychological assessments, early detection methods such as biomarkers, magnetic resonance imaging, and various drugs to reverse the damage from cardiotoxic events are more prevalent and their emphasis has increased tremendously. Fully understanding the mechanisms by which the risk factors action for various patients undergoing cancer treatment is also becoming more prevalent in preventing cardiotoxicity down the line.

Keywords: cardiotoxicity, anticancer therapies, signaling pathway inhibitors, chemotherapy, immunotherapy, cardiotoxicity early detection and prevention

\section{INTRODUCTION}

In the past few decades, anticancer treatment has achieved remarkable progress in improving the quality of life and survival rates of cancer patients. The exploration and application of novel cancer therapeutics have increased tremendously, paralleled with the growth and abundance of literature surrounding the mechanisms underlying cancer metastasis. Novel drug therapies focusing on targeting signaling pathways pertaining to angiogenesis to prevent cellular proliferation via kinase inhibitors have especially been promising. Other methods, including anthracycline chemotherapy, have dramatically improved the outcomes of cancer treatment over the last 10 years (1). However, accompanying with the significant improvements toward cancer treatment, cardiotoxicity-related adverse effects caused by these anticancer therapies, specifically on deleterious cardiovascular effects, such as hypertension, heart failure, QT interval prolongation, and left ventricular dysfunction (LVD) $(2-5)$, as well as heart failure with preserved preserved left ventricular ejection fraction (HFpEF), are increasingly reported (6). The development of cardiotoxicity has also been associated with patient 
age, existing health conditions (at risk of cardiac dysfunction), treatment dosage, and other risk factors (7). Thus, carefully monitoring the development, early detection and prevention of cardiotoxicity, as well as understanding of the interaction between cancer and the cardiovascular system, thereby promoting the development of safer cancer therapeutics, without or with minimized cardiotoxicity, are urgently needed (5).

In this review, we discuss contemporary methods of cancer therapy and the related signaling pathways, which are promoting heart dysfunction and are affected through inhibitory drug treatments that are often compounded with chemotherapy and radiotherapy. We also discuss the importance of the novel therapeutic detection approaches for cardiotoxicity, namely, early detection via biomarkers and cardiovascular imaging, including, but not limited to, magnetic resonance imaging. These alternative treatment routes may provide more insight into the efficacy of cancer treatment strategies and cancer diagnostic tools, which highlight the importance of early detection to avoid later onset of adverse cardiotoxic effects.

\section{CARDIOTOXICITY BY ANTICANCER TREATMENT}

The National Cancer Institute defines cardiotoxicity as "toxicity that affects the heart." Cardiotoxicity may be acute, which occurs during or soon after treatment and is transient, or chronic, and can be categorized into type I (early onset) and type II (late onset) (5). Type I is irreversible cardiac cell injury and usually caused by anthracyclines and chemotherapeutics; type II is typically caused by novel biological-targeted antibodies (8). Chemotherapy and metabolic pathway inhibition has been shown to create adverse side effects, predominantly focusing on myocardial damage and the risks associated with heart failure posttreatment, although the newly emerged targeted drugs such as tyrosine kinase inhibitors and antibodies induce toxicities different from chemotherapy. Often, cardiotoxicity is commonly associated with LVD and other symptoms of systemic heart failure. Furthermore, LVD condition has several facets, which can be related to myocardial toxicity but also to other cardiovascular toxicities, namely, QTc prolongation, arrhythmia, myocardial ischemia induced through atherosclerosis, and pulmonary hypertension. Especially, HFpEF (also called diastolic heart failure) occurs when the lower left ventricle is unable to properly fill with blood during the diastolic phase, and increasingly arises in patients undergoing chemotherapy, and may become predominant HF. Because the pathophysiology underlying HFpEF is heterogeneous, has different phenotypes, and is poorly understood, the etiological definition of HFpEF is variable. Thus, accurate diagnosis is challenging, and currently there is no effective therapy for $\operatorname{HFpEF}(6,9-11)$. However, recently identified novel biomarkers, such as protein biomarker of cardiac stress (ST2), matrix metalloproteinase-2, and growth differentiation factor-15, for the risk stratification of HFpEF may be used for development of significant therapeutic targets for the treatment of HFpEF (12). Furthermore, recent advancements in imaging techniques and exploration into biomarkers have raised the important issue of the multiple comorbidities of cardiotoxicity, many of which are not agglomerated through drug therapies.

Currently, there is no consensus definition of cardiotoxicity. The Cardiac Review and Evaluation Committee of trastuzumabassociated cardiotoxicity defines cardiotoxicity as symptoms of heart failure, decline of left ventricular ejection fraction (LVEF), symptomatic fall in LVEF $\geq 5$ to $<55 \%$ or an asymptomatic reduction of LVEF $\geq 10$ to $<55 \%$ (13). The American Society of Echocardiography and European Association of Cardiovascular Imaging define cardiotoxicity as global longitudinal strain (GLS) with a $10-15 \%$ early reduction (http://asecho.org/wordpress/wpcontent/uploads/2015/12/MLM-Revised-Strain-Code-11-12-15. pdf). The Food and Drug Administration defines LVEF drop $<40-45 \%$ or is $40-49 \%$ with a $\geq 10 \%$ absolute decrease below baseline with anti-HER2 targeted therapy as being necessary to be monitored (14).

The Common Terminology Criteria for Adverse Events (CTCAE) is a descriptive terminology that is used for adverse event reporting based on a grading scale ranging from 1 to 5, with 1 being abnormal elevations in biomarker expression or imaging abnormalities, to 5, death due to cardiotoxicity (CTCAE, version 4.0 National Cancer Institute, June 14, 2010). However, the current reporting scale may be confusing and depends highly on symptomology, which may not represent a clear image of LVD abnormalities, for example, with patients who are asymptomatic. Finally, attributing signs and symptoms of cardiotoxicity prove to be difficult, as many symptoms are may not be induced or attributable to the drug therapy itself. This is especially challenging in older patients, whom often have common comorbidities.

Although the current scale may be deficient in some ways, it is important to address the limitations of potential definitions. Research of this magnitude requires consistency in defining the issue to share a common language and reinforce validity in the research.

\section{CONTEMPORARY THERAPEUTICS FOR NEOPLASIA}

Currently, immunotherapies use the immune system to enhance their antitumor immunity and further immune responses by employing immune checkpoint inhibitors, chimeric antigen receptor (CAR) T cell therapy, and adaptive cell transfer (ACT) (using the patient's own T-cells, engineered to specifically target cancer cells) have been promising in certain cancer treatments (15). Other targeted therapies, specifically focusing on signaling pathway inhibition to prevent certain cell processes from occurring, namely, angiogenesis, also improve clinical outcomes. Angiogenesis, a normal process in which blood vessels are created through currently existing vessels, is a vital process in wound healing, growth, and development. However, growing tumors hijack this process to feed and proliferate tumor cells, thereby creating malignant tumor vessels within the body, making angiogenesis a key factor for tumor growth and survival.

One of the most prominently used and evaluated strategies is the inhibition of the vascular endothelial growth factor (VEGF) signaling cascade. VEGF plays a critical role in angiogenesis 
through binding VEGF receptors and activating the VEGF signaling pathway. Inhibition of angiogenesis, thereby, prevents growing tumors from hijacking the body's natural process (16). Another popular target for cancer therapies is the human epidermal growth factor receptor 2 (HER2). The HER2 protein, also called ERBB2, is commonly overexpressed in patients with breast cancer, which accounts for approximately $15-30 \%$ cases in breast cancer $(17,18)$. Normally, HER2 helps in growth, proliferation, and repairing of abnormal cells within the body. However, similar to the VEGF signaling pathway, tumorigenesis takes control of the cellular process and promotes the proliferation of cancer cells $(19,20)$.

Trastuzumab (Herceptin), a monoclonal antibody, specifically targets HER2. Treatment regimens with Trastuzumab, coupled with chemotherapy, have shown remarkable outcomes in patients with breast cancer after 1 year (21). Anthracycline antibodies, including doxorubicin (DOX), have also been a consistent and prominent form of chemotherapy schemes for nearly half a century. By limiting proliferation of cancer cells via preventative interference with its DNA or RNA structure, DOX is able to halt tumorigenesis and ultimately stop cancer cell proliferation and division $(8,22)$.

\section{TARGETING THE VEGF SIGNALING PATHWAY}

Vascular endothelial growth factor and its corresponding receptors, VEGFR, are one of the most important tyrosine kinase pathways. VEGF includes seven members in its family-VEGF-A, VEGF-B, VEGF-C, VEGF-D, VEGF-E, VEGF-F, and PIGF. VEGF-A is the most representative component. VEGF-A mRNA is expressed in various tissues in the body, such as the lung, kidney, and heart (16). Consequently, the VEGF signaling pathway plays a central role in other signaling pathways which also affect the vasculature, and any alterations to this pathway have been shown to exhibit deleterious effects (23).

Mechanistically, VEGFbinds to three receptor sites-VEGFR-1 (Flt-1), VEGFR-2 (Flk-1), and VEGFR-3 (24). Binding of VEGF to VEGFR-2 initiates the tyrosine signaling cascade, which then facilitates cell migration, proliferation, growth, and vasodilation, all of which are important and contribute to the development of angiogenesis (25). Historically, the VEGF pathway can be targeted using several different methods, including the molecule itself (monoclonal antibodies), receptors (recombinants), or downstream signaling pathways and inhibiting downstream expression (tyrosine kinase inhibition) (4).

Current antiangiogenesis therapies, including the inhibition of VEGF, have shown to have adverse effects on the cardiovascular system $(2,7,16)$. VEGFR-1 and VEGFR-2 are both expressed naturally in endothelial cells (EC) (26). Due to the nature of inhibitory drug therapies, affecting the expression and interaction between VEGF and its receptor sites affects the entire circulatory system and primarily induces proliferation of EC and promoting vascular integrity. Anti-VEGF therapies, such as the tyrosine kinase inhibitor, imatinib, dasatinib, nilotinib, bosutinib, sunitinib, sorafenib, axitinib, and ponatinib, exacerbate LVD, as well as incidences of hypertension, ischemic events, rapid acceleration of atherosclerosis, and other vascular toxic events (27). Inhibition of the VEGF pathway, therefore, can lead to endothelial dysfunction, arising from the disruption of normal endothelial homeostasis mediated through nitric oxide (23).

Meta-analyses with sunitinib have shown increased relative risk for cardiovascular complications. In a meta-analysis of 9,387 patients, the risk of myocardial ischemia caused by sunitinib was 3.03-fold higher compared with placebo (28). Sunitinib has also been speculated to induce LVD risk in over $20 \%$ of patients (28). Ponatinib has been associated with significant cardiovascular toxicity, with an indication of $10 \%$ cardiovascular, $7 \%$ cerebrovascular, and $7 \%$ peripheral adverse events by 28 months after treatment. Hypertension was reported in $26 \%$ of patients treated with this drug (28).

\section{TARGETING THE HER2 SIGNALING PATHWAY}

Trastuzumab is a humanized monoclonal antibody that directly inhibits the HER2 signaling pathway. It is used as first-line therapy with chemotherapy and has been shown to be effective in $25-30 \%$ of breast cancer cases $(5,29)$. However, anti-HER2 therapy was reported to increase the risk for asymptomatic decreased left ventricular ejection fraction, leading to complications such as systemic heart failure. Interestingly, the cardiotoxic effects are shown early (usually within weeks of initial treatment) and are independent of dosing regimens. The cardiotoxic effects are also type II, or reversible, and often not severe in terms of cell death or damage (3).

Mechanistically, Trastuzumab competes for binding sites on the extracellular domain of HER2 and inhibits the activation of the tyrosine kinase signaling pathway $(30,31)$. It has also been speculated that Trastuzumab also induces cell death via antibody treatment, although the data supporting the claim are not yet clear (32). Regardless, HER2 inhibition prevents cell repair and significantly limits proliferation. However, it is speculated that because Trastuzumab's effect is established through targeting the HER2 signaling pathway, which regulates cell differentiation, survival, and repair in healthy tissues, the body's myocytes cannot depend on the repair mechanism either, and that leads to cardiotoxicity when in the presence of anthracyclines (33). Supporting data also come from a study of 179 breast cancer patients, in which $44 \%$ of patients developed a cardiac event (e.g., he art failure or decreased ejection fraction) and a tenth developed a second event $(34,35)$.

\section{ANTHRACYCLINES}

Anthracyclines, a class of chemotherapy drugs, are traditional cancer therapies that have been effective in treating many forms of cancer for the last half century. One of the most prominently used and readily identifiable is the anthracycline DOX. DOXinduced cardiotoxicity is classified as type I, or irreversible. It directly contrasts with Trastuzumab and HER2 inhibition by the fact that cardiotoxicity from DOX is dose dependent (usually doses $\geq 450 \mathrm{mg} / \mathrm{m}^{2}$ ) (36). Moreover, the risk for future 
complications significantly increases with cumulative doses $(37,38)$. Although it was originally speculated that the risks for immediate, on-treatment events decreased with lower doses, it is becoming more apparent that late onset complications can be verified regardless of dose. Therefore, there is no dosage regimen of DOX that is completely safe. The immediate cardiotoxic effects can range from a few weeks to years after treatment, even after the treatment has been discontinued. Incidences and reporting of LVD complications range from 1 to $20 \%$, although the rate may be much higher (39). Some studies estimate that over half of the patients exposed to anthracyclines will develop some forms of LVD within 6 years (40). This claim is further supported by recent studies reporting the incidence of cardiotoxicity in 17.9 and $6.3 \%$ of patients for subclinical and overt cardiotoxicity, respectively, in 9 years (41). Other studies have also shown LVEF in $98 \%$ of patients in a cohort of $9 \%(n=226)$ overall incidence for cardiotoxicity within 1 year after chemotherapy (42).

Chemically, DOX has both hydrophilic and hydrophobic regions, allowing it to bind to both plasma proteins and cell membranes. DOX also has both acidic and base functions, its characteristics of being reoxidized results in the production of reactive oxygen species (ROS) and gives DOX an antineoplastic and antibiotic capabilities $(8,43)$. DOX has several different channels in which it affects the overall homeostasis in the body. One of the most important is its interaction with topoisomerase II. Topoisomerases are isomerase enzymes that participate in the overwinding or underwinding of DNA, allowing DNA to replicate by binding to the double strand DNA and overcome the tangles caused by the double helix. Topoisomerase II has two nuclear localized isoforms: topoisomerase II $\alpha$ and II $\beta$. DOX inhibits topoisomerase interaction with DNA by directly binding to both II $\alpha$ and II $\beta$, forming a DNA cleavage complex that increases double strand breakage $(8,22)$. Although DOX prevents cancer cells from replicating, it also serves as the primary cytotoxic reagent to induce cell apoptosis. DOX affects calcium homeostasis as well. It directly interferes with calcium storage capacity of the mitochondria by activating the selective CsA-sensitive calcium channel, causing calcium overload $(8,44)$ and leads to mitochondrial dysfunction, as well as apoptosis. The decreased calcium levels from the mitochondria have been shown to be irreversible. DOX, therefore, is highly versatile in terms of its uses, as well as its impact on the vasculature, and the cardiovascular system as a whole.

\section{CANCER IMMUNOTHERAPY}

Cancer immunotherapy is a newly emerging treatment method which bases itself on the deeper understanding of the mechanism of antitumor immune responses, discoveries of novel anticancer molecules (peptides and vaccines), and development of innovative technologies of gene transfer (45). Current popular cancer immunotherapy employing inhibitory effects to immune checkpoint receptors has proven to be very effective in several malignancies and has shown very promising clinical outcomes in various types of cancers in the past 10 years. This revolutionized strategy has brought anticancer treatment into a new era $(8,45)$. Anti-cytotoxic T lymphocyte-associated antigen 4 (CTLA-4) and anti-programmed cell death protein-1 (PD-1) have especially been beneficial to ailments, such as melanoma (46). Normally, cancer cells can utilize these receptors to avoid destruction via $\mathrm{T}$ cells in the immune system by binding to CLTA- 4 and reducing naïve $\mathrm{T}$ cell activation or by expressing the cell death protein ligand-1 (PD-L1), which then binds to PD-1 and mediates T cell downregulation and apoptosis. Inhibitors, such as ipilimumab (anti-CTLA-4 monoclonal antibody) and pembrolizumab and nivolumab (anti-PD-1 monoclonal antibodies), bind to and block the inhibitory effects of the receptor sites, thus enhancing the cytotoxic immune response to the cancer cells (45).

Adoptive T cell transfer (ACT) is another novel and a promising method for a wide spectrum of solid cancer treatment. ACT uses a patient's T cells to specifically target tumor cells. Mechanistically, tumor infiltrating lymphocytes (TILs) are collected and genetically engineered into a patient's microenvironment along with systemic interleukin-2 (IL-2) to stimulate their survival and expansion (45). Successful implementation of ACT as a treatment modality for patients with metastatic melanoma has established the basis for multiple modifications and improvements of this strategy for targeting many different cancers (47). It has been reported that in $50 \%$ of patients with metastatic melanoma exhibit objective tumor regression by ACT using autologous TILs (47). As an innate cytokine in the immune system with essential and wide spectrum of immunological functions, IL-2 has long been used for cancer treatment alone and has shown great tumor regression $(48,49)$. IL-2 directly regulates T cell differentiation into different $\mathrm{T}$ cells in response to antigens to enhance the defense of the immune system $(48,49)$. A recent report of a clinical study with patients treated with lymphodepleting conditioning chemotherapy followed by infusion of autologous TILs and high-dose IL-2 showed regression of metastatic uveal melanoma in 7 out of 20 patients, which had been believed to be resistant to immunotherapy previously (50). Similarly, chimeric antigen receptor (CAR)-T therapy has had valuable benefits to certain deficiencies, such as B cell acute lymphoblastic leukemia (B-ALL). Mechanistically, CAR-T functions very similar to ACT, wherein native T-cells are engineered to express a chimeric antigen receptor on the cell membrane. The receptor is coupled with an external binding domain to specifically recognize and bind to tumor antigens. CAR-T also has an internal activation domain that then activates the T-cell once CAR-T binds to its target. In patients with B-ALL, CAR-T therapy is effective in $70-90 \%$ of cases (51).

However, the therapeutic effects are also counterbalanced with similar cardiotoxic effects (52-54). This is especially the case with immunotherapies, since activated $\mathrm{T}$ cell responses may be non-specific to cancer cells, thereby targeting normal tissue as well, leading to frequent immune-related adverse events (IRAEs) such as colitis, endocrinopathies, hepatitis, and pneumonitis. Other events, such as cardiomyopathy, myocardial fibrosis, myocarditis, and acute heart failure, were also reported in single anti-CTLA-4 or anti-PD-1 treatment (54), and lethal myocarditis accompanying with myositis was seen in combinatorial treatment (55), although such cases are rare. Patients using the monoclonal antibody ipilimumab have an IRAE frequency rate of $64-80 \%$ $(54,56,57)$. Patients treated with pembrolizumab have an IRAE 
frequency rate of up to $79 \%(54,58)$. Combination therapies using both ipilimumab and nivolumab have been shown to have tremendous therapeutic efficacy and response rates in patients with melanoma from $19 \%$ using ipilimumab alone to $58 \%$ with the combination $(46,52)$. However, the frequency rate of IRAEs also increased parallel to the combination therapy, reaching as high as $96 \%$ in patients using both ipilimumab and nivolumab (59). CAR-T therapy and ACT both have associated risks with cardiotoxicity. CAR-T therapy, specifically, has links with cytokine release syndrome (CRS), an inflammatory response that is correlated with the activation of CAR-T cells. Manifestations of CRS include arrhythmias, decreased LVEF, and QT prolongation (60).

\section{PREVENTION OF CARDIOTOXICITY AND FUTURE DIRECTIONS}

Reversing certain effects of cancer drug therapies may not always be possible. However, current research into managing and monitoring cardiotoxicity and the various side effects arising from both modern and traditional therapeutics have been very promising. Dexrazoxane, a most promising cardioprotective agent, has been shown to be effective in reducing both acute and chronic cardiotoxicity induced by anthracycline therapy $(8,61)$. Dexrazoxane has been shown to interfere with iron-dependent redox reactions, thereby reducing ROS originating from DOX (62). Dexrazoxane also directly inhibits topoisomerase II $\beta$, thereby preventing anthracycline binding and DNA double strand breaks (63). However, the ability of Dexrazoxane in reducing anthracyclineinduced cardiotoxicity has been limited in its clinical use because of adverse side effects (5). A recent clinical study indicated that Dexrazoxane, in addition to DOX, resulted in higher rates of bone marrow suppression, more febrile neutropenia events and dose reductions (64). Seeking novel protection of cardiotoxicity drugs is thereby more essential and in urgent demand.

Treatments modalities employed either before, after, or before and after cancer therapeutics have proven effective when dealing with certain cardiotoxic effect risk factors, such as heart failure. The use of beta blockers (BBs), angiotensinconverting enzyme (ACE) inhibitor, angiotensin inhibitors, and mineralocorticoid receptor antagonists have all shown promising results in preventing cardiac damage and is an active ongoing investigation area (7). BBs have antioxidant properties and were utilized for chemotherapy-induced LVEF and other systolic LV dysfunction. One of the new generation BBs and a most commonly used agent, carvedilol, showed strong antioxidant characteristics and greater protective effect on anthracycline-induced cardiomyopathy $(6,8)$. A recent report demonstrated that favorable effects of ACE inhibitors and BBs on preventing cardiotoxicity and improving survival of breast cancer patients treated with trastuzumab and/or anthracyclines (65). Moreover, beta-adrenergic blockade with nebivolol, metoprolol, lisinopril, etc., has also been proven to be effective for cardiomyopathy $(66,67)$. However, reports on some BBs demonstrating beneficial cardioprotective effects in patients with LVEF remains controversial (68). Novel modalities, such as lcz696 (valsartan/sacubitril) $(69,70)$ and ivabradine can also be used to treat heart failure and subsequent impaired myocardial systolic dysfunction $(6,71)$. Furthermore, the cardioprotective role of ranolazine (inhibition of late INa elevation) and phosphodiesterase-5 inhibitors has been reported (66). In addition, nutrition supplements and exercise have shown positive effects on cardiomyopathy (66). However, significant supportive clinical data are still needed to prove the protective function of these novel agents in patients affected by cardiotoxic effects (7).

The role of biomarkers has also been important in early detection. Elevated or abnormal expression levels of several biomarkers can be used as indicators for screening and assess the risk factors for future cardiotoxicity complications. Interleukin-6 (IL-6), a cytokine produced by adipose tissues, increases blood pressure and induces inflammation. Overexpression of IL-6 inhibits cell apoptosis, stimulates angiogenesis, and plays a role in drug resistance (72). There have also been promising data exploring biomarkers that pertain to type I and type II cardiac events. Increases in troponin-I, brain-type natriuretic peptide (BNP), and N-terminal-pro-BNP have all been linked to drops in $\operatorname{LVEF}(5,73)$. Plasma myeloperoxidase also predicts a decrease in cardiac function (7). MicroRNA has emerged as a potential marker for early onset of heart failure. miR-1, miR-133b, and miR-146a were all upregulated corresponding to DOX chemotherapy (74).

Early detection is vital when considering the deleterious effects that drug therapeutics may have. One facet in which early detection has been outlined is through the risk factors for both cardiovascular diseases and cancer. Shared risk factors, such as obesity, carcinogenic agent usage (e.g., tobacco, smoking, and alcohol), previous history of diabetes, hypertension, non-modifiable risk factors (e.g., age and sex), and physical activity, should all be taken into consideration, especially when pertaining to drug therapeutics, thereby minimalizing the risk of future cardiotoxicity events (75).

Current exploration into psychological stress as an additional risk factor is promising (76). The mechanism in which psychological distress plays in cardiovascular dysfunction is twofold, focusing on behavioral and pathophysiological. In terms of behavioral mechanisms, it is best understood that personality types, temperament, anxiety, and depression can influence and are related to other risk factors, such as unhealthy lifestyles, lack of exercise, smoking, and alcohol consumption (77). In terms of pathophysiological mechanisms, being under psychological distress may form complications such as platelet dysfunction, autonomic nervous system dysregulation, hypothalamic-pituitaryadrenal axis (HPA-axis) dysregulation, cellular aging, and inflammatory activation. Platelet activation has shown increases in patients with depression (78). The HPA-axis has a known role and association in cardiovascular dysfunction via cortisol regulation (79). Patients with depression, anxiety, and fatigue often show elevated cortisol levels (80). Patients with depression and anxiety also have shorter telomere length, a biomarker for cellular aging and also an associated risk factor for cardiovascular dysfunction (81).

Takotsubo cardiomyopathy (TC), known as stress cardiomyopathy or broken heart syndrome, may be caused by emotional stress and long-term anxiety (82). TC shows similar images as 
that of acute coronary syndrome and is characterized by dynamic electrocardiographic changes along with transient, severe, and reversible LVD, but unclear pathophysiological mechanisms $(82,83)$. It is more common in elder women and was taken into account only in recent years in cancer patients treated with anticancer drugs suggesting that chemotherapy can induce TC (83-85).

Detection and early screening of cardiotoxicity via imaging techniques have become more prevalent. Two-dimensional echocardiography (2DE) has been the standard for quite some time. However, assessment of LVEF and GLS via three-dimensional echocardiography (3DE) and speckle-tracking echocardiography, respectively, has been shown to be a valuable asset in early detection and is able to overcome many challenges that affect traditional 2DE methods $(7,86)$. Moreover, GLS seems to predate LVEF decreases, and impairment of GLS in HEpEF has been reported (87). Thus, GLS seems a better indicator of myocardial dysfunction prior heart failure progression. Cardiac magnetic resonance imaging has emerged in recent years and is used as the gold standard parameter. It is accurate, reproductive, and reliable and has higher sensitivity than $2 \mathrm{DE}$ and $3 \mathrm{DE}$ in detecting early changes in global and regional cardiac function, and its high contrast-to-noise ratio provides excellent structural characteristics $(88,89)$.

\section{CONCLUSION}

Cardiotoxicity is one of the most deleterious effects arising from cancer therapeutics and a major barrier to survivorship. However, today's cancer patients should not be tomorrow's cardiovascular disease patients. Recent research has shed the light of optimism

\section{REFERENCES}

1. McGowan JV, Chung R, Maulik A, Piotrowska I, Walker JM, Yellon DM. Anthracycline chemotherapy and cardiotoxicity. Cardiovasc Drugs Ther (2017) 31:63-75. doi:10.1007/s10557-016-6711-0

2. Moslehi JJ. Cardiovascular toxic effects of targeted cancer therapies. $N$ Engl J Med (2016) 375:1457-67. doi:10.1056/NEJMra1100265

3. Domercant J, Polin N, Jahangir E. Cardio-oncology: a focused review of anthracycline-, human epidermal growth factor receptor 2 inhibitor-, and radiation-induced cardiotoxicity and management. Ochsner J (2016) 16:250-6.

4. Abdel-Qadir H, Ethier JL, Lee DS, Thavendiranathan P, Amir E. Cardiovascular toxicity of angiogenesis inhibitors in treatment of malignancy: a systematic review and meta-analysis. Cancer Treat Rev (2017) 53:120-7. doi:10.1016/j. ctrv.2016.12.002

5. Jain D, Russell RR, Schwartz RG, Panjrath GS, Aronow W. Cardiac complications of cancer therapy: pathophysiology, identification, prevention, treatment, and future directions. Curr Cardiol Rep (2017) 19:36. doi:10.1007/ s11886-017-0846-x

6. Ponikowski P, Voors AA, Anker SD, Bueno H, Cleland JG, Coats AJ, et al. ESC guidelines for the diagnosis and treatment of acute and chronic heart failure: the task force for the diagnosis and treatment of acute and chronic heart failure of the European Society Of Cardiology (ESC) developed with the special contribution of the Heart Failure Association (HFA) of the ESC. Eur Heart J (2016) 37:2129-200. doi:10.1093/eurheartj/ehw128

7. Tromp J, Steggink LC, Van Veldhuisen DJ, Gietema JA, van der Meer P. Cardio-oncology: progress in diagnosis and treatment of cardiac dysfunction. Clin Pharmacol Ther (2017) 101:481-90. doi:10.1002/cpt.614

8. Tocchetti CG, Cadeddu C, Di Lisi D, Femmino S, Madonna R, Mele D, et al. From molecular mechanisms to clinical management of antineoplastic with an emphasis on early prevention. Continued research and discussion will further advance our literature pertaining to cardiotoxicity, opening up additional avenues for safer treatment strategies. Further investigation into alternative therapeutics, as well as the increasing information and understanding of modern technologies in screening and detecting at-risk patients will be helpful in developing and evaluating different modalities for cancer therapeutics. Moreover, such research will be able to fill in the gaps in understanding cardiotoxicity and explore other avenues of research that are often overlooked.

Understanding the importance of risk factors and associated risks, such as the role of psychological distress, are necessary to advance the care of cancer patients. Minimizing the risk of cardiovascular complications induced through various therapies is vital to treatment and care. This, again, is an emphasis on the importance of early detection and risk assessment when considering drug administration to certain patients.

\section{AUTHOR CONTRIBUTIONS}

JD and HC wrote the manuscript and finalized the manuscript.

\section{FUNDING}

This work was supported by NIH Grants R01HL-093242, R01HL118676, and P20 RR018758, Oklahoma Center for Advanced Science Technology (OCAST) Grant HR09-116, Established Investigator Award from the American Heart Association (AHA), and Department of Defense Grant W81XWH-11-1-00226 to HC; JD was supported by the Fleming Scholar Program in Oklahoma Medical Research Foundation.

drug-induced cardiovascular toxicity: a translational overview. Antioxid Redox Signal (2017). doi:10.1089/ars.2016.6930

9. Senni M, Greene SJ, Butler J, Fonarow GC, Gheorghiade M. Drug development for heart failure with preserved ejection fraction: what pieces are missing from the puzzle? Can J Cardiol (2017) 33:768-76. doi:10.1016/j.cjca.2017.03.013

10. Oren $\mathrm{O}$, Goldberg S. Heart failure with preserved ejection fraction: diagnosis and management. Am JMed (2017) 130:510-6. doi:10.1016/j. amjmed.2016.12.031

11. Lewis GA, Schelbert EB, Williams SG, Cunnington C, Ahmed F, McDonagh TA, et al. Biological phenotypes of heart failure with preserved ejection fraction. JAm Coll Cardiol (2017) 70:2186-200. doi:10.1016/j.jacc.2017. 09.006

12. Cypen J, Ahmad T, Testani JM, DeVore AD. Novel biomarkers for the risk stratification of heart failure with preserved ejection fraction. Curr Heart Fail Rep (2017) 14:434-43. doi:10.1007/s11897-017-0358-4

13. Seidman A, Hudis C, Pierri MK, Shak S, Paton V, Ashby M, et al. Cardiac dysfunction in the trastuzumab clinical trials experience. J Clin Oncol (2002) 20:1215-21. doi:10.1200/JCO.2002.20.5.1215

14. Kondapalli L. Cardiotoxicity: An Unexpected Consequence of HER2-Targeted Therapies. (2016). Available from: http://www.acc.org/latest-in-cardiology/ articles/2016/06/06/09/32/cardiotoxicity

15. Wang DY, Okoye GD, Neilan TG, Johnson DB, Moslehi JJ. Cardiovascular toxicities associated with cancer immunotherapies. Curr Cardiol Rep (2017) 19:21. doi:10.1007/s11886-017-0835-0

16. Di Lisi D, Madonna R, Zito C, Bronte E, Badalamenti G, Parrella P, et al. Anticancer therapy-induced vascular toxicity: VEGF inhibition and beyond. Int J Cardiol (2017) 227:11-7. doi:10.1016/j.ijcard.2016.11.174

17. Burstein HJ. The distinctive nature of HER2-positive breast cancers. N Engl J Med (2005) 353:1652-4. doi:10.1056/NEJMp058197 
18. Mitri Z, Constantine T, O'Regan R. The HER2 receptor in breast cancer: pathophysiology, clinical use, and new advances in therapy. Chemother Res Pract (2012) 2012:743193. doi:10.1155/2012/743193

19. Dempsey KS. Chemotherapy-induced cardiotoxicity in women. Crit Care Nurs Clin North Am (2008) 20:343-50. doi:10.1016/j.ccell.2008.03.004

20. Dempsey KS. Chemotherapy-induced cardiotoxicity in women. Heart Fail Clin (2011) 7:427-35. doi:10.1016/j.hfc.2011.04.004

21. Gianni L, Dafni U, Gelber RD, Azambuja E, Muehlbauer S, Goldhirsch A, et al. Treatment with trastuzumab for 1 year after adjuvant chemotherapy in patients with HER2-positive early breast cancer: a 4-year follow-up of a randomised controlled trial. Lancet Oncol (2011) 12:236-44. doi:10.1016/ S1470-2045(11)70033-X

22. Swift LP, Cutts SM, Nudelman A, Levovich I, Rephaeli A, Phillips DR. The cardio-protecting agent and topoisomerase II catalytic inhibitor sobuzoxane enhances doxorubicin-DNA adduct mediated cytotoxicity. Cancer Chemother Pharmacol (2008) 61:739-49. doi:10.1007/s00280-007-0528-2

23. Kamba T, McDonald DM. Mechanisms of adverse effects of anti-VEGF therapy for cancer. Br J Cancer (2007) 96:1788-95. doi:10.1038/sj.bjc.6603813

24. Ferrara N, Gerber HP, LeCouter J. The biology of VEGF and its receptors. Nat Med (2003) 9:669-76. doi:10.1038/nm0603-669

25. Koch S, Tugues S, Li X, Gualandi L, Claesson-Welsh L. Signal transduction by vascular endothelial growth factor receptors. Biochem J (2011) 437:169-83. doi:10.1042/BJ20110301

26. Morbidelli L, Donnini S, Ziche M. Targeting endothelial cell metabolism for cardio-protection from the toxicity of antitumor agents. Cardiooncology (2016) 2:3. doi:10.1186/s40959-016-0010-6

27. Damrongwatanasuk R, Fradley MG. Cardiovascular complications of targeted therapies for chronic myeloid leukemia. Curr Treat Options Cardiovasc Med (2017) 19:24. doi:10.1007/s11936-017-0524-8

28. Pun SC, Neilan TG. Cardiovascular side effects of small molecule therapies for cancer. Eur Heart J (2016) 37:2742-5. doi:10.1093/eurheartj/ehw361

29. Chen ZI, Ai DI. Cardiotoxicity associated with targeted cancer therapies. Mol Clin Oncol (2016) 4:675-81. doi:10.3892/mco.2016.800

30. Albanell J, Bellmunt J, Molina R, Garcia M, Caragol I, Bermejo B, et al. Nodenegative breast cancers with p53(-)/HER2-neu(-) status may identify women with very good prognosis. Anticancer Res (1996) 16:1027-32.

31. Molinaro M, Ameri P, Marone G, Petretta M, Abete P, Di Lisa F, et al. Recent advances on pathophysiology, diagnostic and therapeutic insights in cardiac dysfunction induced by antineoplastic drugs. Biomed Res Int (2015) 2015:138148. doi:10.1155/2015/138148

32. Molina MA, Codony-Servat J, Albanell J, Rojo F, Arribas J, Baselga J. Trastuzumab (herceptin), a humanized anti-Her2 receptor monoclonal antibody, inhibits basal and activated Her2 ectodomain cleavage in breast cancer cells. Cancer Res (2001) 61(12):4744-9.

33. Bartsch R, Wenzel C, Steger GG. Trastuzumab in the management of early and advanced stage breast cancer. Biologics (2007) 1:19-31.

34. Walls GM, Lyon AR, Harbinson MT, Hanna GG. Cardiotoxicity following cancer treatment. Ulster Med J (2017) 86:3-9.

35. Farolfi A, Melegari E, Aquilina M, Scarpi E, Ibrahim T, Maltoni R, et al. Trastuzumab-induced cardiotoxicity in early breast cancer patients: a retrospective study of possible risk and protective factors. Heart (2013) 99:634-9. doi:10.1136/heartjnl-2012-303151

36. De Angelis A, Urbanek K, Cappetta D, Piegari E, Pia Ciuffreda L, Rivellino A, et al. Doxorubicin cardiotoxicity and target cells: a broader perspective. Cardiooncology (2016) 2:2. doi:10.1186/s40959-016-0012-4

37. Lipshultz SE, Lipsitz SR, Sallan SE, Dalton VM, Mone SM, Gelber RD, et al. Chronic progressive cardiac dysfunction years after doxorubicin therapy for childhood acute lymphoblastic leukemia. JClin Oncol (2005) 23:2629-36. doi:10.1200/JCO.2005.12.121

38. Manrique CR, Park M, Tiwari N, Plana JC, Garcia MJ. Diagnostic strategies for early recognition of cancer therapeutics-related cardiac dysfunction. Clin Med Insights Cardiol(2017) 11:1179546817697983. doi:10.1177/1179546817697983

39. Cautela J, Lalevee N, Ammar C, Ederhy S, Peyrol M, Debourdeau P, et al. Management and research in cancer treatment-related cardiovascular toxicity: challenges and perspectives. Int J Cardiol (2016) 224:366-75. doi:10.1016/j. ijcard.2016.09.046

40. Swain SM, Whaley FS, Ewer MS. Congestive heart failure in patients treated with doxorubicin: a retrospective analysis of three trials. Cancer (2003) 97:2869-79. doi:10.1002/cncr.11407
41. Lotrionte M, Biondi-Zoccai G, Abbate A, Lanzetta G, D’Ascenzo F, Malavasi V, et al. Review and meta-analysis of incidence and clinical predictors of anthracycline cardiotoxicity. Am J Cardiol (2013) 112:1980-4. doi:10.1016/j. amjcard.2013.08.026

42. Cardinale D, Colombo A, Bacchiani G, Tedeschi I, Meroni CA, Veglia F, et al. Early detection of anthracycline cardiotoxicity and improvement with heart failure therapy. Circulation (2015) 131:1981-8. doi:10.1161/ CIRCULATIONAHA.114.013777

43. Mitry MA, Edwards JG. Doxorubicin induced heart failure: phenotype and molecular mechanisms. Int JCardiol Heart Vasc (2016) 10:17-24. doi:10.1016/j.ijcha.2015.11.004

44. Zhou S, Starkov A, Froberg MK, Leino RL, Wallace KB. Cumulative and irreversible cardiac mitochondrial dysfunction induced by doxorubicin. Cancer Res (2001) 61:771-7.

45. Papaioannou NE, Beniata OV, Vitsos P, Tsitsilonis O, Samara P. Harnessing the immune system to improve cancer therapy. Ann Transl Med (2016) 4:261. doi:10.21037/atm.2016.04.01

46. Ledford H. Cocktails for cancer with a measure of immunotherapy. Nature (2016) 532:162-4. doi:10.1038/532162a

47. Rosenberg SA, Restifo NP, Yang JC, Morgan RA, Dudley ME. Adoptive cell transfer: a clinical path to effective cancer immunotherapy. Nat Rev Cancer (2008) 8:299-308. doi:10.1038/nrc2355

48. Bright R, Coventry BJ, Eardley-Harris N, Briggs N. Clinical response rates from interleukin-2 therapy for metastatic melanoma over 30 years' experience: a meta-analysis of 3312 patients. J Immunother (2017) 40:21-30. doi:10.1097/ CJI.0000000000000149

49. Jiang T, Zhou C, Ren S. Role of IL-2 in cancer immunotherapy. Oncoimmunology (2016) 5:e1163462. doi:10.1080/2162402X.2016.1163462

50. Chandran SS, Somerville RPT, Yang JC, Sherry RM, Klebanoff CA, Goff SL, et al. Treatment of metastatic uveal melanoma with adoptive transfer of tumour-infiltrating lymphocytes: a single-centre, two-stage, single-arm, phase 2 study. Lancet Oncol (2017) 18:792-802. doi:10.1016/S1470-2045(17)30251-6

51. Jackson HJ, Rafiq S, Brentjens RJ. Driving CAR T-cells forward. Nat Rev Clin Oncol (2016) 13:370-83. doi:10.1038/nrclinonc.2016.36

52. Cheng F, Loscalzo J. Autoimmune cardiotoxicity of cancer immunotherapy. Trends Immunol (2017) 38:77-8. doi:10.1016/j.it.2016.11.007

53. Jain V, Bahia J, Mohebtash M, Barac A. Cardiovascular complications associated with novel cancer immunotherapies. Curr Treat Options Cardiovasc Med (2017) 19:36. doi:10.1007/s11936-017-0532-8

54. Heinzerling L, Ott PA, Hodi FS, Husain AN, Tajmir-Riahi A, Tawbi H, et al. Cardiotoxicity associated with CTLA4 and PD1 blocking immunotherapy. J Immunother Cancer (2016) 4:50. doi:10.1186/s40425-016-0152-y

55. Johnson DB, Balko JM, Compton ML, Chalkias S, Gorham J, Xu Y, et al. Fulminant myocarditis with combination immune checkpoint blockade. $N$ Engl J Med (2016) 375:1749-55. doi:10.1056/NEJMoa1609214

56. Graziani G, Tentori L, Navarra P. Ipilimumab: a novel immunostimulatory monoclonal antibody for the treatment of cancer. Pharmacol Res (2012) 65:9-22. doi:10.1016/j.phrs.2011.09.002

57. Hodi FS, O'Day SJ, McDermott DF, Weber RW, Sosman JA, Haanen JB, et al. Improved survival with ipilimumab in patients with metastatic melanoma. $N$ Engl J Med (2010) 363:711-23. doi:10.1056/NEJMoa1003466

58. Hamid O, Robert C, Daud A, Hodi FS, Hwu WJ, Kefford R, et al. Safety and tumor responses with lambrolizumab (anti-PD-1) in melanoma. N Engl J Med (2013) 369:134-44. doi:10.1056/NEJMoa1305133

59. Larkin J, Chiarion-Sileni V, Gonzalez R, Grob JJ, Cowey CL, Lao CD, et al. Combined nivolumab and ipilimumab or monotherapy in untreated melanoma. N Engl J Med (2015) 373:23-34. doi:10.1056/NEJMoa1504030

60. Davila ML, Riviere I, Wang X, Bartido S, Park J, Curran K, et al. Efficacy and toxicity management of 19-28z CAR T cell therapy in B cell acute lymphoblastic leukemia. Sci Transl Med (2014) 6:224ra25. doi:10.1126/scitranslmed.3008226

61. Minotti G, Menna P, Salvatorelli E, Cairo G, Gianni L. Anthracyclines: molecular advances and pharmacologic developments in antitumor activity and cardiotoxicity. Pharmacol Rev (2004) 56:185-229. doi:10.1124/pr.56.2.6

62. Hochster HS. Clinical pharmacology of dexrazoxane. Semin Oncol (1998) 25:37-42.

63. Lyu YL, Kerrigan JE, Lin CP, Azarova AM, Tsai YC, Ban Y, et al. Topoisomerase IIbeta mediated DNA double-strand breaks: implications in doxorubicin cardiotoxicity and prevention by dexrazoxane. Cancer Res (2007) 67:8839-46. doi:10.1158/0008-5472.CAN-07-1649 
64. Tahover E, Segal A, Isacson R, Rosengarten O, Grenader T, Gips M, et al. Dexrazoxane added to doxorubicin-based adjuvant chemotherapy of breast cancer: a retrospective cohort study with a comparative analysis of toxicity and survival. Anticancer Drugs (2017) 28:787-94. doi:10.1097/CAD. 0000000000000514

65. Wittayanukorn S, Qian J, Westrick SC, Billor N, Johnson B, Hansen RA. Prevention of trastuzumab and anthracycline-induced cardiotoxicity using angiotensin-converting enzyme inhibitors or beta-blockers in older adults with breast cancer. Am J Clin Oncol (2017). doi:10.1097/COC.0000000000000389

66. Cadeddu C, Mercurio V, Spallarossa P, Nodari S, Triggiani M, Monte I, et al. Preventing antiblastic drug-related cardiomyopathy: old and new therapeutic strategies. J Cardiovasc Med (Hagerstown) (2016) 17:e64-75. doi:10.2459/ JCM.0000000000000382

67. Nohria A. $\beta$-Adrenergic blockade for anthracycline- and trastuzumabinduced cardiotoxicity: is prevention better than cure? Circ Heart Fail (2013) 6:358-61. doi:10.1161/CIRCHEARTFAILURE.113.000267

68. Pun SC, Neilan TG. Cardioprotective Interventions: Where Are We? (2016). Available from: http://www.acc.org/latest-in-cardiology/articles/2016/07/21/ 07/28/cardioprotective-intervetions

69. Ayalasomayajula S, Langenickel T, Pal P, Boggarapu S, Sunkara G. Clinical pharmacokinetics of sacubitril/valsartan (LCZ696): a novel angiotensin receptor-neprilysin inhibitor. Clin Pharmacokinet (2017) 56:1461-78. doi:10.1007/s40262-017-0543-3

70. Lin LM, Wu Y, Wu MF, Lin JX. Focus on the novel cardiovascular drug LZC696: from evidence to clinical consideration. Cardiovasc Drugs Ther (2016) 30:623-33. doi:10.1007/s10557-016-6699-5

71. Swedberg K, Komajda M, Bohm M, Borer JS, Ford I, Dubost-Brama A, et al. Ivabradine and outcomes in chronic heart failure (SHIFT): a randomised placebo-controlled study. Lancet (2010) 376:875-85. doi:10.1016/ S0140-6736(10)61198-1

72. Guo Y, Xu F, Lu T, Duan Z, Zhang Z. Interleukin-6 signaling pathway in targeted therapy for cancer. Cancer Treat Rev (2012) 38:904-10. doi:10.1016/j. ctrv.2012.04.007

73. Cardinale D, Sandri MT, Martinoni A, Tricca A, Civelli M, Lamantia G, et al. Left ventricular dysfunction predicted by early troponin I release after high-dose chemotherapy. J Am Coll Cardiol (2000) 36:517-22. doi:10.1016/ S0735-1097(00)00748-8

74. Cappetta D, Rossi F, Piegari E, Quaini F, Berrino L, Urbanek K, et al. Doxorubicin targets multiple players: a new view of an old problem. Pharmacol Res (2018) 127:4-14. doi:10.1016/j.phrs.2017.03.016

75. Koene RJ, Prizment AE, Blaes A, Konety SH. Shared risk factors in cardiovascular disease and cancer. Circulation (2016) 133:1104-14. doi:10.1161/ CIRCULATIONAHA.115.020406

76. Schoormans D, Rottmann N, Pedersen SS, van de Poll-Franse L. Cardiovascular co-morbidity in cancer patients: the role of psychological distress. Cardiooncology (2016) 2:9. doi:10.1186/s40959-016-0019-x

77. Colleoni M, Mandala M, Peruzzotti G, Robertson C, Bredart A, Goldhirsch A. Depression and degree of acceptance of adjuvant cytotoxic drugs. Lancet (2000) 356:1326-7. doi:10.1016/S0140-6736(00)02821-X

78. Musselman DL, Marzec UM, Manatunga A, Penna S, Reemsnyder A, Knight BT, et al. Platelet reactivity in depressed patients treated with paroxetine: preliminary findings. Arch Gen Psychiatry (2000) 57:875-82. doi:10.1001/ archpsyc.57.9.875

79. Molloy GJ, Perkins-Porras L, Strike PC, Steptoe A. Type-D personality and cortisol in survivors of acute coronary syndrome. Psychosom Med (2008) 70:863-8. doi:10.1097/PSY.0b013e3181842e0c

80. Burke HM, Davis MC, Otte C, Mohr DC. Depression and cortisol responses to psychological stress: a meta-analysis. Psychoneuroendocrinology (2005) 30:846-56. doi:10.1016/j.psyneuen.2005.02.010

81. Verhoeven JE, Revesz D, Epel ES, Lin J, Wolkowitz OM, Penninx BW. Major depressive disorder and accelerated cellular aging: results from a large psychiatric cohort study. Mol Psychiatry (2014) 19:895-901. doi:10.1038/ mp.2013.151

82. Yoshikawa T. Takotsubo cardiomyopathy, a new concept of cardiomyopathy: clinical features and pathophysiology. Int J Cardiol (2015) 182:297-303. doi:10.1016/j.ijcard.2014.12.116

83. Fernandez SF, Basra M, Canty JMJ. Takotsubo cardiomyopathy following initial chemotherapy presenting with syncope and cardiogenic shock a case report and literature review. J Clin Exp Cardiol (2011) 2:124. doi:10.4172/2155-9880.1000124

84. Coen M, Rigamonti F, Roth A, Koessler T. Chemotherapy-induced Takotsubo cardiomyopathy, a case report and review of the literature. BMC Cancer (2017) 17:394. doi:10.1186/s12885-017-3384-4

85. Munoz E, Iliescu G, Vejpongsa P, Charitakis K, Karimzad K, Lopez-Mattei J, et al. Takotsubo stress cardiomyopathy: "good news" in cancer patients? J Am College Cardiol (2016) 68:1143-4. doi:10.1016/j.jacc.2016.06.027

86. Swiger KJ, Singh J, Lenihan DJ. Cardiomyopathic toxicity from chemotherapy: is there an opportunity for preemptive intervention? Curr Treat Options Cardiovasc Med (2017) 19:20. doi:10.1007/s11936-017-0517-7

87. Carluccio E, Biagioli P, Alunni G, Murrone A, Leonelli V, Pantano P, et al. Advantages of deformation indices over systolic velocities in assessment of longitudinal systolic function in patients with heart failure and normal ejection fraction. Eur J Heart Fail (2011) 13:292-302. doi:10.1093/ eurjhf/hfq203

88. Moonen M, Oury C, Lancellotti P. Cardiac imaging: multimodality advances and surveillance strategies in detection of cardiotoxicity. Curr Oncol Rep (2017) 19:63. doi:10.1007/s11912-017-0622-5

89. Avelar E, Strickland CR, Rosito G. Role of imaging in cardio-oncology. Curr Treat Options Cardiovasc Med (2017) 19:46. doi:10.1007/s11936-017-0546-2

Conflict of Interest Statement: The authors declare that the research was conducted in the absence of any commercial or financial relationships that could be construed as a potential conflict of interest.

The reviewer AF and handling editor declared their shared affiliation.

Copyright $(2018$ Dong and Chen. This is an open-access article distributed under the terms of the Creative Commons Attribution License (CC BY). The use, distribution or reproduction in other forums is permitted, provided the original author(s) and the copyright owner are credited and that the original publication in this journal is cited, in accordance with accepted academic practice. No use, distribution or reproduction is permitted which does not comply with these terms. 